\title{
Escobar, Mónica (2016). "La enseñanza de la Matemática en aulas plurigrado. Un estudio de caso sobre un Instituto Superior de Formación Docente de la provincia de Buenos Aires". Tesis de Maestría en Educación. Facultad de Humanidades y Ciencias de la Educación. UNLP
}

\author{
Elisa Marchese \\ elilaca@hotmail.com \\ Universidad Nacional de La Plata, Argentina \\ Rufina Bonadeo \\ bonadeorufina@hotmail.com \\ Universidad Nacional de La Plata, Argentina
}

\begin{abstract}
Cita sugerida: Marchese, E. y Bonadeo, R. (2020). [Revisión de la tesis La enseñanza de la Matemática en aulas plurigrado. Un estudio de caso sobre un Instituto Superior de Formación Docente de la provincia de Buenos Aires por M. Escobar]. Archivos de Ciencias de la Educación, 14(17), e084. https://doi.org/10.24215/23468866e084
\end{abstract}

La tesis "La enseñanza de la Matemática en aulas plurigrado. Un estudio de caso sobre un Instituto Superior de Formación Docente de la provincia de Buenos Aires" ${ }^{1}$ es el resultado de la investigación realizada por la Prof. Mónica Escobar para obtener el título de Magister en Educación. La misma versa sobre la formación inicial de docentes en la enseñanza de la matemática que se desempeñarán en escuelas rurales con plurigrado. Para ello, indaga en torno a las prácticas formativas docentes que tienen lugar en un Instituto Superior de Formación Docente (ISFD) de la provincia de Buenos Aires, que son interpeladas y complejizadas por otros dispositivos y le permiten recuperar de un modo específico, las prácticas de la enseñanza de la matemática en los contextos de ruralidad.

Si bien en una primera instancia la investigación pretendía responder a la pregunta ¿Cómo se enseña matemática en aulas plurigrado de escuelas primarias rurales unitarias?, luego de dialogar con los estudios preliminares sobre el tema elegido y entablar contacto con iniciativas en un ISFD, el interrogante se amplió para analizarlo desde la formación docente inicial. Así, la nueva pregunta pasó a ser ¿cómo se enseña y cómo 
se aprende a enseñar matemática en aulas plurigrado de escuelas primarias rurales unitarias? Esta pregunta recorre toda su obra, la cual se compone por una introducción, cinco capítulos, referencias bibliográficas y dos anexos.

La introducción se divide en dos partes, la Presentación del Problema y el Plan de Exposición, en los cuales presenta la estructura general de la tesis y los capítulos que componen su investigación.

En la presentación del problema, la información recuperada permite reconocer que la educación rural se trata de un espacio heterogéneo que alberga y agrupa diversas realidades. Identifica que en la formación docente inicial y continua se ofrecen escasas reflexiones para quienes se desempeñan en estos espacios como docentes, quienes en su formación abordan contenidos que refieren a situaciones de enseñanza en escuelas graduadas de sección única. Es por ello que, como se indica en muchos casos, los y las docentes de las escuelas rurales planifican y piensan la enseñanza apelando a distintos saberes docentes que se adaptan o inventan en la práctica y/o se difunden entre pares, dando lugar así a lo que Terigi denomina la "invención del hacer".

Por su parte, el capítulo 1 lleva por título La educación rural desde los marcos legales y curriculares, la formación inicial y las condiciones del trabajo docente. En el mismo, a través de un abordaje histórico con énfasis en el relevamiento de normativas producidas por los diferentes organismos de gestión y definición de la política educativa, se reconstruye el origen de la escuela primaria, del plurigrado y de la formación docente en el país, subrayando el contexto de la provincia de Buenos Aires. También se incluye un apartado donde se presentan experiencias similares con este tipo de propuestas en países como Perú, México y Uruguay.

En el capítulo 2, Antecedentes y marco teórico metodológico, la autora realiza, en primer lugar, un exhaustivo relevamiento de estudios e investigaciones que abordan el objeto de estudio delimitado en la tesis, para en un segundo momento presentar el problema y las preguntas de investigación, como así también, el punto de partida desde el que fueron formuladas. Las mismas se encuentran agrupadas en preguntas sobre la formación docente vinculada a la enseñanza de la matemática, y preguntas que están relacionadas con la enseñanza de la matemática en aquellas instituciones donde realizan las prácticas los y las docentes en formación.

Seguidamente, se expone el marco teórico el cual expresa el posicionamiento elegido para la construcción del trabajo de campo. Se encuentra organizado en diversas perspectivas conceptuales que permiten abordar el problema de investigación recuperando los aportes de la didáctica de la matemática con énfasis en la Teoría de Situaciones, la Teoría de la Transposición Didáctica de Chevallard, la Teoría de los Campos Conceptuales, las interacciones en la clase de matemática entre estudiantes y docentes y los aportes que caracterizan el contexto rural y la enseñanza en plurigrado, como así también aquellas investigaciones sobre la formación docente inicial.

Finalmente se presenta la estrategia metodológica utilizada, inscribiendo la tesis en un proyecto de investigación más amplio denominado "Un estudio descriptivo sobre la enseñanza de la matemática en aulas plurigrados en escuelas rurales unitarias desde la mirada de la formación docente” considerando el caso de un ISFD de la provincia de Buenos Aires.

La selección del caso responde a un interés por la propuesta académica que ofrece el instituto seleccionado ya que es poco habitual la consideración de contenidos vinculados al plurigrado en la formación docente inicial. En el trabajo de campo se desarrollan entrevistas individuales y grupales a docentes del instituto y a docentes que reciben practicantes en escuelas rurales con plurigrado. Asimismo, selecciona tres escuelas primarias rurales con plurigrado para realizar observaciones de las clases de matemática a cargo de los y las docentes de la institución.

Ya en el capítulo 3, El contexto rural y la enseñanza en plurigrado en la formación docente, se presenta el análisis de las entrevistas, explicitando la línea de análisis y las argumentaciones provenientes de éstas en relación a por qué incluir o desestimar el contenido de la educación rural y la enseñanza en plurigrado en la formación docente inicial. Seguidamente, se exponen instancias de formación docente continua en las que se aborda la temática del plurigrado y se destina un apartado especial a las preguntas realizadas a los y las docentes 
que se desempeñan en contextos rurales y que se convierten en fuente de consulta valiosa para profesores/ as del ISFD.

El capítulo 4, La tarea de los maestros a cargo de escuelas rurales unitarias, se divide en dos partes: por un lado, el relevamiento de las tareas que desempeñan los y las docentes de las escuelas rurales unitarias, los contextos y las condiciones en las que se inscribe su desempeño profesional. Mientras que, la segunda parte, refiere a la enseñanza de la matemática en las escuelas primarias rurales con plurigrado donde se propone un recorrido por la didáctica de la matemática, recuperando la planificación de las clases y las observaciones realizadas en el trabajo de campo.

En el último capítulo, De los supuestos y preguntas iniciales a los hallazgos y nuevos interrogantes, se presentan las conclusiones del trabajo de investigación, donde se arriba a la idea de que las temáticas vinculadas a la educación en contextos de ruralidad en la formación de docentes es mínima, aunque descubre que los y las formadores utilizan principalmente dos estrategias de búsqueda de información: bibliografía sobre la temática y el saber experto de los/as maestros/as rurales. Respecto a esta segunda fuente de consulta, se advierte sobre la idealización y naturalización que suele generarse alrededor de estas experiencias y se propone analizar y reflexionar sobre las mismas para que se instituyan en puntos de partida para la producción de nuevos conocimientos.

En relación a las observaciones de las clases de matemática, se identifica que los y las docentes que participaron en la investigación generan entre los y las estudiantes espacios de producción colectiva de conocimiento. Mientras que, en relación a la tarea docente en escuelas rurales unitarias, se recogen las preocupaciones que tienen los y las docentes que se desempeñan en estos espacios, que abarcan desde las condiciones del trabajo docente (multiplicidad de tareas, aislamiento, soledad en el trabajo) hasta preocupaciones por la tarea de la enseñanza en plurigrado como las planificaciones y la organización del trabajo en el aula.

Asimismo, se recupera de un modo positivo, la interacción que se generó entre formadores/as y maestros/as orientadores/as durante el proceso de reflexión sobre las prácticas áulicas y formativas usuales y la exploración de alternativas, otorgándole al instituto valiosos tópicos para incluir en la formación docente inicial de los y las estudiantes del profesorado. En este aspecto, la investigación suma algunos supuestos de partida referidos más precisamente a la enseñanza de la matemática en el aula plurigrado, que plantean la predominancia de propuestas de trabajo individual y la atención casi personalizada de docentes a cada estudiante, en especial a quienes asisten a los primeros años, generando cierta autonomía en los años superiores. Esta organización responde a la gradualidad propia del aula estándar que, sumada a la disposición graduada del Diseño Curricular del Nivel, dificulta la elaboración de planificaciones y ordenamientos de espacios de trabajo colectivo que involucren a la totalidad del estudiantado.

De este modo, el estudio del caso seleccionado le permitió poner en duda algunos supuestos que la autora tenía al inicio de la investigación. Tanto las iniciativas del instituto de incluir la enseñanza en plurigrado en contextos rurales en la formación docente inicial, como las propuestas de cátedra vinculadas a la residencia docente y la oferta de espacios de definición institucional sobre Educación Rural, pusieron en cuestionamiento el supuesto de que la mayoría de los ISFD no abordan la especificidad del contexto rural y la enseñanza en plurigrado. En segundo lugar, esta investigación permitió corroborar la escasa bibliografía que aborda y problematiza la temática del plurigrado. Esto último fue motivo para impulsar la propuesta de un taller de escritura colectiva que establece como propósito sistematizar nuevos aportes sobre la enseñanza en aulas plurigrado que sirvan como material de consulta para el instituto concomitante o para quienes lo requieran. El mismo está integrado por docentes de escuelas rurales, profesoras y autoridades del ISFD dónde tuvo lugar la investigación, la autora de la tesis y docentes y estudiantes de Ciencias de la Educación de la FaHCE-UNLP con interés en profundizar en el abordaje de temáticas vinculadas a la educación en contextos de ruralidad. 
Así, la tesis permite generar nuevos interrogantes en torno a la formación docente inicial para quienes se van a desempeñar en otros niveles educativos que no necesariamente sea el primario, a la vez que visibiliza la necesidad de que la formación docente continua recupere este tipo de temáticas como contenidos de la capacitación docente. También, deja abierta la posibilidad de pensar la enseñanza de otras áreas además de la matemática, desde el aporte de las didácticas específicas.

Sin lugar a dudas, esta tesis se configura como punto de referencia en el campo de estudios sobre la enseñanza, el cual se nutre y complejiza con preguntas que provienen del campo de la formación docente, de la didáctica de la matemática y de la educación en plurigrado. Este cruce de perspectivas permite identificar un área de vacancia en el abordaje de la educación en plurigrado, que le devuelve nuevas preguntas y nuevos hallazgos, abriendo posibilidades para futuros trabajos.

\section{Notas}

1 Disponible en: http://www.memoria.fahce.unlp.edu.ar/tesis/te.1330/te.1330.pdf

2 Terigi, Flavia (2008). Organización de la enseñanza en los plurigrados de escuelas rurales. (Tesis de Maestría). Buenos Aires: FLACSO, Argentina. Recuperado de

https://repositorio.flacsoandes.edu.ec/bitstream/10469/1266/1/TFLACSO-2009FZT.pdf 